\title{
Dyslipidemia and Its Relationship with DifferentAnthropometric Measures in Nigerian Adults
}

\author{
Okpara Ihunanya Chinyere ${ }^{1}$, Adediran Olufemi Sola ${ }^{1}$ \\ 1. Department of Internal Medicine, P. M. B. 102119, Benue State University Makurdi, Nigeria.
}

\begin{abstract}
:
Background: Dyslipidemia is a known risk factor for atherosclerotic cardiovascular disease and it appears that regional fat distribution plays a role in determining the type of dyslipidemia present in an individual. The present study aims at determining the relationship between dyslipidemia and anthropometric measures in an adult Nigerian population.
\end{abstract}

Method: In a cross-sectional community based study, 229 subjects were selected by a stratified random sampling method. Body mass index (BMI), Waist Circumference (WC), and Waist to height ratio (W/Ht) were the anthropometric indices measured to determine their relationship with lipid profile which include total cholesterol(TC), triglyceride(TG), high density lipoprotein cholesterol(HDL-C), and low density lipoprotein cholesterol $(L D L-C)$.

Results: Anthropometric measures showed no correlations with lipid parameters but however correlated with systolic and diastolic blood pressures with WC having the strongest correlation.The overall prevalence of dyslipidemia in the study population was $58.1 \%$. The pattern of dyslipidemia was as follows: $42.8 \%$ had low $H D L-C, 21.4 \%$ had elevated TG, $2.6 \%$ had elevated TC and $1.7 \%$ had elevated LDL-C.

Conclusion: Anthropometric measures can predict hypertension better than dyslipidemia. There is a high prevalence of dyslipidemia in Nigeria and this is mainly due to low HDL-C and elevated TG.

Key words: Anthropometric measure, blood pressure, dyslipidemia.

\section{Introduction}

Dyslipidemia is one of the several risk factors identified for cardiovascular diseases (CVD) ${ }^{[1]}$. Other risk factors include hypertension, obesity, diabetes mellitus, sedentary life style and cigarette smoking. Among the above risk factors, lipids have been widely investigated due to their extensive association with atherosclerotic plaque formation inside the arteries as well as vessel stenosis. It is for this reason that dyslipidemia has been introduced as an important risk factor for atherosclerosis and $\mathrm{CVD}^{[2,3]}$.

Obesity has proven to be an important risk factor for CVD and is strongly associated with CVD risk factors ${ }^{[4,5]}$. Most studies use anthropometric measures to measure adiposity. Waist Circumference (WC), Waist to hip ratio (WHR) and Waist to height ratio (W/Ht) have all been used as measures of central obesity (where visceral adipose tissue is stored), and body mass index (BMI) measured as weight in kilograms divided the square of the height in meters has been used as a measure of generalized obesity ${ }^{[6]}$. Studies that analysed associations between anthropometric measures and abdominal/visceral fat measured with computed tomography reported WC to be a better measure of central obesity ${ }^{[6-9]}$.

Early studies reported that excess trunk fat was associated with high plasma triglyceride (TG) levels ${ }^{[10,11]}$. More recently others have also shown that abdominal obesity was related to high plasma TG levels ${ }^{[12-14]}$. It has also been reported that regional distribution of body fat, especially excessive deposition of abdominal fat, was associated with low concentrations of serum high density lipoprotein cholesterol (HDL-C) and this association was stronger in men than women ${ }^{[15]}$. An Iranian study has shown that WC and W/Ht ratio showed greater correlation with total cholesterol (TC),TG, low density lipoprotein cholesterol (LDL-C), TC/HDL-C level than did BMI ${ }^{[16]}$. BMI neither gives an indication for the relationship between fat and fat - free mass, nor does it indicate fat distribution and for a given BMI, physically fit individuals have less total and abdominal fat, compared to unfit individuals ${ }^{[17]}$.

The result of a study carried out in African women has demonstrated the association of abdominal obesity with high TG, low HDL-C and high LDL-C ${ }^{[18]}$. Studies carried out in Nigeria were mainly on prevalence and pattern of dyslipidemia and all revealed high prevalence rates with low HDL-C being the commonest dyslipidemia ${ }^{[19-21]}$.

The aim of this study was to find out which anthropometric measure of adiposity best correlates with dyslipidemia and its pattern and prevalence in an adult Nigerian population. 


\section{Materials And Method}

In a cross-sectional community based study, by a stratified random sampling method, 229 subjects aged $18-78$ years were recruited. Stratification was by gender and location. 113 subjects were recruited from the urban area called Garki-village in Abuja - Abuja Municipal Area Council of Nigeria, while 116 came from a rural area called Kuseki. Both areas are within the Federal Capital Territory (FCT) in Nigeria. The study was carried out between May 2009 and June 2010.

Ethical approval was obtained from the department of Health, Abuja Municipal area council and the ethical committee of the Benue state University Makurdi. Informed consent in written form or by a thumb print was obtained from the participants after due explanation before they were used for the study.

A structured questionnaire including designed to acquire information regarding age, gender, marital status, level of education and location was administered to the participants or completed on their behalf. Anthropometric data which included weight, height and WC were obtained. The weight was measured to the nearest $0.5 \mathrm{~kg}$ using a weighing scale with the participants wearing light clothing and removing their foot wears.

Height was measured to the nearest $0.5 \mathrm{~cm}$ using a stadiometer. The body mass index was calculated as weight in kilograms divided by the square of the height in meters ${ }^{[22]}$. The WC was measured at midpoint between the lower border of the ribcage and iliac crestsusing a flexible tape, and passing along the umbilical level of the unclothed abdomen at the end of normal expiration. W/Ht ratio was calculated by dividing the WC by the height.

Venous blood samples were collected after 12 hours overnight fast. TC, HDL-C, TG and fasting blood glucose were determined using an automatic analyser (Hitachi 7060, Hitachi Tokyo Japan). LDL-C was calculated using Friedewaldformula ${ }^{[23]}$.

Three blood pressure readings were taken in the sitting position at five minute intervals after five minutes rest in the morning according to the seventh report of the Joint National Committee on the Prevention, Detection, Evaluation and Treatment of High Blood Pressure (JNC7) ${ }^{[24]}$. The systolic blood pressure (SBP) and the diastolic blood (DBP) were the first and fifth koroktoff sounds respectively. The first reading was discarded and the average of the last two measurements used for data analysis. Hypertension was defined as $\mathrm{SBP} \geq 140 \mathrm{mmHg}$ and $/$ or $\mathrm{DBP} \geq 90 \mathrm{mmHg}$.

The diagnosis of dyslipidemia was based on the National Cholesterol Education Program Adult Treatment Panel III criteria (NCEP ATPIII) ${ }^{[25]}$.

The data was analysed using the statistical packages for social sciences (SPSS) version 20 software. For continuous variables means and standard deviations were calculated and the means compared using the independent samples t-test. Pearson Chi-Square test was used to compare proportions. The Pearson Correlation Coefficient test was used to analyse the relationship between anthropometric indices and CVD risk factors. Values of $p$ below 0.05 were considered statistically significant.

\subsection{Demographic characteristics of the study population \\ III. Results}

The total number of participants in the study was 229 . Males were 113 in number while females were 116 in number. A total of 113 of the participants came from the urban area while 116 came from the rural area.

The mean age of the males was $43.04 \pm 14.36$ while that of the females was $42.09 \pm 14.36$. There was no significant difference in the mean age of the participants by locality and gender. However the mean weight, BMI, WC, SBP, DBP were significantly higher in the urban dwellers than rural dwellers $(p<0.0001)$. Males were significantly taller than females $(p<0.001)$ while females had significantly higher BMI than males $(p=$ 0.002). These are shown in TABLES1 and 2. About half of the population had no education or just primary education $(58.1 \%)$ while a large percentage of them $(79 \%)$ were married.

\subsection{Prevalence of CVD risk factors/ pattern of Dyslipidemia in the study population.}

Out of the 229 subjects who participated in the study, 133 had dyslipidemia and 96 had no dyslipidemia. The overall prevalence of dyslipidemia in the study group was $58.1 \%$. It was more prevalent in the rural than urban area with rates of $59.5 \%$ versus $56.6 \%$ but the difference was not statistically significant $\left(\chi^{2}\right.$ $=0.190, p=0.663)$. Dyslipidemia was more prevalent in females than males with rates of $53.4 \%$ versus $46.6 \%$ but the difference was not statistically significant $\left(\chi^{2}=0.945, \mathrm{p}=0.331\right)$.

The pattern of dyslipidemia in the total study population was as follows: $1.7 \%$ had elevated LDL-C, $2.6 \%$ had elevated TC, $21.4 \%$ had elevated TG and $42.8 \%$ had low HDL-C. This is shown in fig. 1 . The pattern of dyslipidemia by locality was as follows: in the urban area, $0.4 \%$ had elevated LDL-C, $1.3 \%$ had elevated TC, $13.5 \%$ had elevated TG and $18.3 \%$ had low HDL-C. In the rural area, $1.3 \%$ had elevated LDL-C, $1.3 \%$ had elevated TC, $7.9 \%$ had elevated TG and $24.5 \%$ had low HDL-C.This is shown in fig. 2. With respect to gender: in males, $3.5 \%$ had elevated LDL-C, $3.5 \%$ had elevated TC, $22.1 \%$ had elevated TG, and 36.3\% had low HDL- 
C. In females, $0.0 \%$ had elevated LDL-C, $1.7 \%$ had elevated TC, $20.7 \%$ had elevated TG, and $49.1 \%$ had low HDL-C.This is shown in fig. 3.

The mean age, BMI,WC,W/Ht, DBP and FBG were higher in the dyslipidemia than in the no dyslipidemia group but the difference was not statistically significant. However the mean TG, LDL-C, HDL-C, and SBP were significantly higher in the dyslipidemia than no dyslipidemia group. These are shown in TABLE 3.

The mean SBP in the urban dwellers was $127.13 \pm 23.84 \mathrm{mmHg}$ while that in the rural dwellers was $113.98 \pm 16.89 \mathrm{mmHg}(\mathrm{p}<0.001)$. The mean DBP in the urban dwellers was $79.42 \pm 15.16 \mathrm{mmHg}$ while that in the rural dwellers was $74.26 \pm 9.90 \mathrm{mmHg}(\mathrm{p}=0.003)$. This is shown in TABLE 1 . The prevalence of hypertension in the study group was $22.7 \%$ with a higher prevalence in the urban $(32.7 \%)$ than rural $(12.9 \%)$ dwellers $(\mathrm{p}<0.001)$.

The prevalence of central obesity in the study population was $20.1 \%$ while that of generalized obesity was $10.0 \%$. The overall prevalence of obesity whether by central or generalized was $22.3 \%$. It was more prevalent in the urban than rural area with rates of $40.7 \%$ versus $4.3 \%$ respectively $(\mathrm{p}<0.001)$. Obesity was also more prevalent in females than males with rates of $36.2 \%$ versus $8.0 \%(\mathrm{p}<0.001)$ respectively. The prevalence of central obesity in females was $34.5 \%$ and in males was $5.3 \%(p<0.001)$. The prevalence of generalized obesity was $15.5 \%$ in females and $4.4 \%$ in females $(\mathrm{p}<0.001)$.

\subsection{Relationship between anthropometric measures and dyslipidemia.}

Anthropometric measures showed no correlations with lipid parameters but however correlated significantly with systolic and diastolic blood pressures with WC having the strongest correlation. These are shown in TABLE 4.

\section{Discussion}

The present study has demonstrated that anthropometric measures showed no correlations with lipid parameters but rather correlated significantly with systolic and diastolic blood pressures with WC having the strongest correlation. It has also demonstrated a high prevalence of dyslipidemia in the study population with low HDL-C and high TG being the commonest dyslipidemia. Interestingly in our study, dyslipidemia was more prevalent in the rural than in the urban area. It was also more prevalent in females than males.

The findings of our study differ from that of Chehrei et.al. ${ }^{[16]}$ in 2007 who found that WHR and WC were better indices than BMI for predicting dyslipidemia in Iranian adults. The results of some studies showed that BMI cannot differentiate the fat from the muscle mass and also cannot indicate adipose tissue distribution ${ }^{[17]}$. Therefore some researchers believe that BMI is not a good indicator of adiposity to predict CVD risk factors ${ }^{[26]}$. Also VanDijket. al. ${ }^{[27]}$ who conducted a meta-analysis on different anthropometric measures and their association with CVD risk factors found WC to have the strongest association amongst almost all CVD risk factors for both men and women although not always statistically significant.

Our study showed that WC had the strongest correlation amongst anthropometric measures with SBP and DBP.

The lack of correlation between anthropometric measures and lipid parameters in our study may be due to the sample size. A larger sample size may have demonstrated significant correlation especially with WC as shown in other studies. The significant correlation between anthropometric measures and SBP and DBP suggests that there is a direct relationship between obesity and blood pressure ${ }^{[28]}$. Hypertension prevalence increases linearly with obesity prevalence.

Our study showed an overall high prevalence of obesity ( $22.3 \%$ ) and the prevalence of central obesity was also high $(20.1 \%)$ compared to generalized obesity which was $10 \%$. From this study one can deduce that the high prevalence of hypertension in the study group is due to obesity. With the unrelenting epidemic of obesity, more than $50 \%$ of all cases of hypertension can be attributed to obesity ${ }^{[29]}$.

The prevalence of dyslipidemia in our study was high at $58.1 \%$ and this was mainly contributed by low HDL-C and elevated TG as shown in fig. 1. This finding supports that found in other studies carried out in Nigeria where they found that low HDL-C is the commonest dyslipidemia in Nigeria ${ }^{[19-21]}$. Note also that the higher prevalence ofdyslipidemia in females was mainly due to low HDL-C as TG was higher in males than females and that central obesity was more prevalent in females than males. This finding differs from that of Despreset $\mathrm{a}^{15}$. who found excess deposition of abdominal fat in association with low HDL-C to be a stronger association in males than females. Interestingly low HDL-C and elevated TG are the lipid parameters used in the definition of the metabolic syndrome ${ }^{[30]}$ which is a syndrome that involves an overall increase in all-cause mortality and CVD death ${ }^{[25]}$. Janssen et. al. ${ }^{[31]}$ analysed the data from the National Health and Nutrition Examination Survey (NHANESIII) database to determine whether the prevalence of CVD risk factors is greater in individuals with high WC values compared with normal WC values with the same BMI category. Individuals with higher WC values were reported to have significantly greater prevalence of CVD risk factors even with in the same BMI category thus underscoring the importance of WC. Little wonder WC is the anthropometric 
measure used in the definition of the metabolic syndrome. When examining the cluster of CVD risk factors in the metabolic syndrome, Schneider et.al. ${ }^{[32]}$ and Dervanx et. al. ${ }^{[33]}$ concluded that WC had stronger association with the metabolic syndrome.

The general idea behind using anthropometric adiposity measures is to predict a certain risk and to measure a certain change over time when comparing interventions. Considering the high prevalence of central obesity in this study which is a risk factor for hypertension and dyslipidemia all leading to the metabolic syndrome, health interventions such exercises to induce weight loss will be highly beneficial. Velthiuset.al ${ }^{[34]}$ conducted a randomised controlled trial and investigated the effect of a 12 month moderate to vigorous exercise programme combining effects of aerobic and muscle strength training on body composition among 189 sedentary, postmenopausal women. Their data showed that the exercise programme was able to reduce fat mass, increase lean body mass and reduce WC, although weight and BMI were not affected. Any significant reduction in WC will reduce CVD risk factors.

\section{Conclusion And Recommendation}

From the evidence available, anthropometric measures predict hypertension better than dyslipidemia. There is a high prevalence of dyslipidemia, obesity and hypertension in our Nigerian environment. All this is pointing to an increase in the prevalence of the metabolic syndrome and CVD mortality. Exercises and other measures to reduce obesity should be encouraged.

References

[1]. Bamba V, Rader DJ. Obesity and atherogenicdyslipidemia. Gastroenterology. 2007; 132: $2181-2190$.

[2]. Krause MP, Hallage T, Gama MP, Sasaki JE Miculis CP, Buzzacheia CF et al. Association between lipid profile and adiposity in women over 60. Arq Bras Cardiol. 2007; 89 (3) : $147-149$.

[3]. Sarnis RS, De Souza FI, Schoeps DO, Catherino P, De Oliveira MC, Pessotti CF, et al. Relationship between waist circumference and nutritional status, lipid profile and blood pressure in low socio-economic level pre-school children. Arq Bras Cardiol. 2006; 87 (2) : $153-158$.

[4]. ATP III. Third report of the National Cholesterol Education Program (NCEP) Expert Panel on Detection, Evaluation, and Treatment of High Blood Cholesterol in Adults ( Adult Treatment Panel III). Final Report 2002, Bethesda, Maryland USA : National Institutes of Health.

[5]. Fogelholm M. Physical activity, fitness and fatness : relations to mortality, morbidity and disease risk factors. A systematic review. Obes Rev 2009.

[6]. Molarius A, Seidell JC. Selection of anthropometric indicators for classification of abdominal fatness - a critical review. Int J ObesMetabdisord. 1998; 22 (8) $719-727$.

[7]. Pouliot MC, Depres JP, Lemieux S, et al. Waist circumference and abdominal sagittal diameter : best simple anthropometric indexes of abdominal visceral adipose tissue accumulation and related cardiovascular risk in men and women. Am J Cardiol. 1994; $73(7): 460-468$.

[8]. Hill JO, Sidney S, Lewis CE, et al. Racial differences in amounts of visceral adipose tissue in young adults : the CARDIA ( Coronary Artery Risk Development in Young Adults) study. Am J ClinNutr. 1999; 69 (3) : $381-387$.

[9]. Vazquez G, Duval S, Jacobs DR Jr et al. Comparison of body mass index, waist circumference and waist/hip ratio in predicting incident diabetes : a meta - analysis. Epidemiol Rev. 2007; $29: 115$ - 128.

[10]. Albrink MJ, Melga JW. Interrelationship between skinfold thickness, serum lipids and blood sugar in normal men. Am J ClinNutr $1964 ; 15: 255-261$.

[11]. Allard C, Goulet C. Serum Lipids : An epidemiological study of an active Montreal population. Can Med Assoc J 1968 ; 98 : 627 637.

[12]. Kissebah AH, Vydellingum N, Murray R, et al. Relation of body fat distribution to metabolic complications of obesity. J ClinEndocrinolMetab 1982; $54: 254-260$

[13]. Krotklewski M, Bjorntorp P, Sjostrom L, Smith U. Impact of obesity on metabolism in men and women. Importance of regional adipose tissue distribution. J Clin Invest 1983; $72: 1150-1162$.

[14]. Evans DJ, Hoffman RG, KalhoffRK, Kissebah AH. Relationship of body fat topography to insulin sensitivity and metabolic profiles in premenopausal women. Metabolism $1984 ; 33 ; 68-75$.

[15]. Despres JP, Allard C, Tremblay A, Talbot J, Bouchard C. Evidence for a regional component of body fats in the association with serum lipids in men and women. Metabolism 1985; $34: 967-973$.

[16]. Chehrei A, Sadrnia S, Keshteli AH, Daneshmand MA, Rezaei J. Correlation of dyslipidemia with waist to height ratio, waist circumference and body mass index in Iranian adults. Asia Pac J ClinNutr. 2007; 16 (2) : $248-253$.

[17]. Romeo - Corral A, Somers VK, Sierra - Johnson J et al. Accuracy of body mass index in diagnosing obesity in the adult general population. Int J Obes (Lond) 2008; 32 (6): 959 - 966.

[18]. Crowther NJ, Ferros WF, Ojwang PJ, Rheeder P. The effect of abdominal obesity on insulin sensitivity and serum lipid and cytokine concentrations African women. ClinEndocrinol (Oxf) 2006; $64:(5): 535-541$.

[19]. Odenigbo CU, Oguejiofor OC, Odenigbo UM, Ibeh CC, Ajaero CM, Odike MA. Prevalence of dyslipidemia in apparently healthy professionals in Asaba, South-South Nigeria. NigClinPract 2008; 11:330 - 335.

[20]. Sani MU, Wahab KW, Yusuf BO, Gbadamosi M, Johnson OU, Gbadamosi A. Modifiable cardiovascular risk factors among apparently healthy Nigerian population - a cross sectional study. BMC research notes 2010; 3: 11.

[21]. Osuji CU, Nzerem BA, Meludu S, Dioka CE, Nwobodo CE, Amilo GI. The prevalence of overweight/obesity and dyslipidemia amongst a group of women attending 'August' meeting. Niger Med J 2010; 51: $155-159$.

[22]. Quetelet AD. Physique De L'Homme, quoted by Pengelly CDR. Body mass index and abdominal girth in the diagnosis of obesity. Proc. R Col. Physicians, Edinburg 1994; $24: 174-180$.

[23]. Frieldwald WT, Levy RI, Friedrickson DS. Estimation of the concentration of low - density lipoprotein in plasma, without use of the preparative ultracentrifuge. Clin Chem. 1972; 18: $499-502$. 
[24]. Chobanian AV, Ba Kris GL, Black HR, Cushman WC, Green LA et al. Seventh report of the Joint National Committee on Prevention, Detection, Evaluation, and Treatment of High Blood Pressure. Hypertension 2003; 12 (6) : 1206 - 1252.

[25]. Executive summary of the third report of National Cholesterol Education Program ,expert panel on detection, evaluation and treatment of high blood cholesterol in adults ( adult treatment panel - III of National Cholesterol Education Program. JAMA 2001; $285: 2486-2497$.

[26]. WHO. IASO. IOTF. The Asia - Pacific Perspective. Redefining Obesity and its treatment. In WHO editor. Obesity : preventing and managing the global epidemic. Geneva WHO; 2000.

[27]. VanDijk SB, Takken T, Prinsen EC, Wittink H. Different anthropometric adiposity measures and their association with cardiovascular risk factors : a meta - analysis. Neth Heart J 2012; 20 (5) : $208-218$

[28]. Kadiri S, Walker O, Salako BL et al. Blood pressure, hypertension and its correlates in urbanized workers in Ibadan, Nigeria : a revisit. J Hums Hypertens $1999 ; 13: 23-27$.

[29]. Victor RG, Kaplan MN. Systemic Hypertension: Mechanisms and Diagnosis : in Saunders Elsevier (eds),Braunwald's heart disease : a text book of cardiovascular medicine. $8^{\text {th }}$ edition (Pennsylvania : Philadelphia 2008) $1027-1048$.

[30]. Galassi A, Reynolds K, He J. Metabolic syndrome and risk of cardiovascular disease : a meta - analysis. Am J Med. 2006 ; 119 (10): $812-819$.

[31]. Janssen I, Katzmarzyk PT, Ross R. Body mass index, Waist circumference, and health risk : evidence in support of current nati onal Institutes of Health guide lines. Arch Intern Med. 2002: 162 (18) : 2074 - 2079.

[32]. Schneider HJ, Glasemer H, Klotsche J et al. Accuracy of anthropometric indicators of obesity to predict cardiovascular risk. J Clin EndocrinolMetab . 2007; 92 (2) : $589-594$.

[33]. Dervaux N, Wubuli M, Megnein JL et al. Comparative associations of adiposity measures with cardiometabolic risk burden in asymptomatic subjects. Atherosclerosis, 2008; $201(2): 413-417$.

[34]. Velthius MJ, Kremers SP, Candel MJ, et al. Exercise programme affects body composition but not weight in postmenopausal women. Menopause 2009; 16 (4):777-784.

Tables and Figures

Table 1- Characteristics of the study population by locality

\begin{tabular}{|l|l|l|l|l|}
\hline Variable & Urbann =113Mean( SD ) & Ruraln = 116Mean( SD ) & t-test & p-value \\
\hline Age & $43.24(13.76)$ & $41.91(15.08)$ & 0.70 & 0.485 \\
\hline Weight & $69.43(13.61)$ & $58.98(8.29)$ & 7.04 & $<0.001^{*}$ \\
\hline Height & $1.59(0.08)$ & $1.61(0.07)$ & -1.70 & 0.090 \\
\hline BMI & $27.35(4.96)$ & $22.72(2.50)$ & 8.95 & $<0.001^{*}$ \\
\hline WC & $90.06(13.83)$ & $80.49(7.26)$ & 6.58 & $<0.001^{*}$ \\
\hline SBP & $127.13(23.84)$ & $113.98(16.89)$ & 4.83 & $<0.001^{*}$ \\
\hline DBP & $79.42(15.16)$ & $74.26(9.90)$ & 3.06 & $0.003^{*}$ \\
\hline
\end{tabular}

*=statistically significant, $B M I=$ Body mass index

$W C=$ Waist circumference, $S B P=$ Systolic blood pressure

$D B P=$ Diastolic blood pressure.

Table 2- Characteristics of the study population by gender

\begin{tabular}{|l|l|l|l|l|}
\hline \multicolumn{1}{|c|}{ Variable } & $\begin{array}{l}\text { Male } \\
\mathbf{n = 1 1 3} \\
\text { Mean(SD) }\end{array}$ & $\begin{array}{l}\text { Female } \\
\text { n=116 } \\
\text { Mean(SD) }\end{array}$ & t-test & p-value \\
\hline Age & $43.04(14.54)$ & $42.09(14.46)$ & 0.50 & 0.620 \\
\hline Weight & $63.95(9.05)$ & $64.32(14.93)$ & -0.22 & 0.825 \\
\hline Height & $1.63(0.06)$ & $1.57(0.07)$ & 6.11 & $<0.001^{*}$ \\
\hline BMI & $24.07(3.17)$ & $25.91(5.43)$ & -3.11 & $0.002^{*}$ \\
\hline WC & $83.75(11.34)$ & $86.64(12.44)$ & -1.84 & 0.068 \\
\hline SBP & $120.34(19.78)$ & $120.60(23.32)$ & -0.09 & 0.926 \\
\hline DBP & $78.27(11.72)$ & $75.38(14.04)$ & 1.69 & 0.093 \\
\hline
\end{tabular}

*= statistically significant, BMI = Body mass index

$W C=$ Waist circumference, $S B P=$ Systolic blood pressure

$D B P=$ Diastolic blood pressure.

Table 3 CVD risk factors in the Dyslipidemia versus No Dyslipidemia subjects

\begin{tabular}{|l|l|l|l|l|}
\hline Variable & DyslipidemiaMean (SD) & No Dyslipidemia Mean (SD) & t - test & p- value \\
\hline Age & $43.46(14.84)$ & $41.13(13.75)$ & 1.20 & 0.229 \\
\hline BMI & $25.14(5.07)$ & $24.80(3.70)$ & 0.55 & 0.583 \\
\hline WC & $85.90(11.64)$ & $84.26(12.41)$ & 1.03 & 0.305 \\
\hline W/Ht & $53.76(7.30)$ & $52.66(8.34)$ & 1.07 & 0.287 \\
\hline TC & $3.26(0.99)$ & $3.26(0.89)$ & 0.00 & 0.999 \\
\hline TG & $1.60(0.44)$ & $1.30(0.22)$ & 6.09 & $0.000^{*}$ \\
\hline HDL-C & $1.21(0.40)$ & $1.57(0.32)$ & -7.17 & $0.000^{*}$ \\
\hline LDL-C & $1.32(0.82)$ & $1.09(0.79)$ & 2.04 & $0.043^{*}$ \\
\hline FBG & $66.76(16.04)$ & $65.45(21.41)$ & 0.53 & 0.598 \\
\hline SBP & $123.17(21.29)$ & $116.72(21.57)$ & 2.24 & $0.026^{*}$ \\
\hline DBP & $76.99(12.15)$ & $76.56(14.14)$ & 0.25 & 0.086 \\
\hline
\end{tabular}




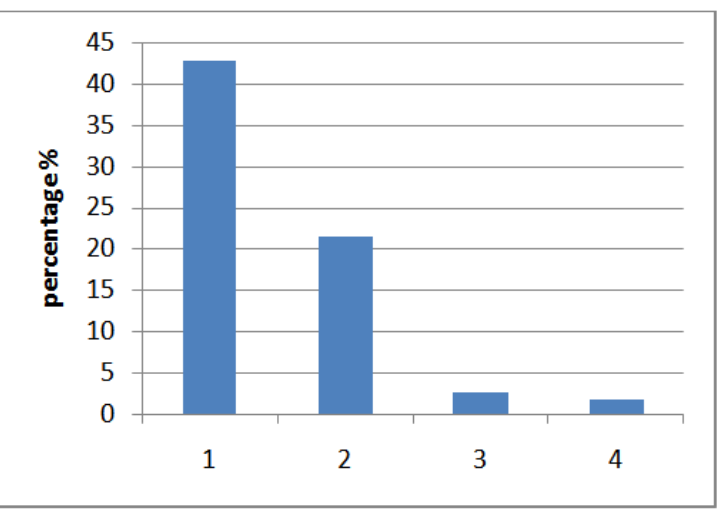

Figure 1- Pattern of Dyslipidemia in the participants $1=$ low HDL-C; 2 = high TG; 3 = high TC; $4=$ high LDL-C

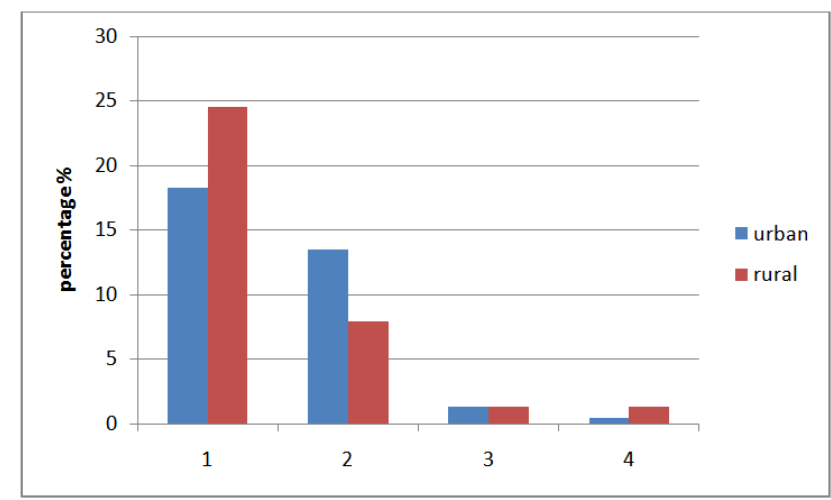

Figure 2 - Pattern of dyslipidemia by locality $1=$ low HDL-C; 2 = high TG; $3=$ high TC; $4=$ high LDL-C

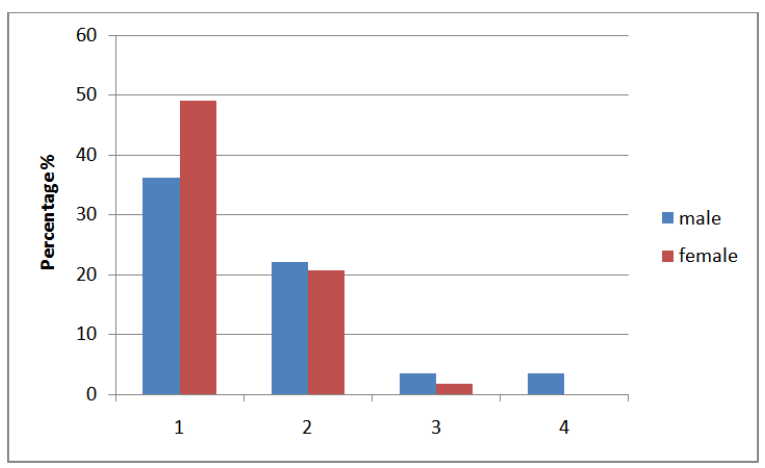

Figure 3 - Pattern of dyslipidemia by gender $1=$ low HDL-C; $2=$ high TG; $3=$ high TC; $4=$ high LDL-C

Table 2Relationship between Anthropometric indices and CVD risk factors.

\begin{tabular}{|l|l|l|l|}
\hline Variables & $\begin{array}{l}\text { BMI } \\
\text { r(p-value) }\end{array}$ & $\begin{array}{l}\text { WC } \\
\text { r(p-value) }\end{array}$ & $\begin{array}{l}\text { W/Ht } \\
\text { r(p-value) }\end{array}$ \\
\cline { 2 - 5 } Age & $-0.022(0.742)$ & $0.133(0.044)^{*}$ & $0.186(0.005)^{*}$ \\
\hline TC & $-0.062(0.347)$ & $-0.032(0.631)$ & $-0.053(0.472)$ \\
\hline LDL-C & $-0.073(0.273)$ & $-0.059(0.374)$ & $-0.106(0.109)$ \\
\hline TG & $0.048(0.474)$ & $0.117(0.076)$ & $0.077(0.248)$ \\
\hline HDL-C & $0.022(0.743)$ & $0.022(0.744)$ & $0.060(0.366)$ \\
\cline { 2 - 5 } FBG & $0.066(0.320)$ & $0.005(0.935)$ & $0.015(0.816)$ \\
\hline SBP & $0.400(<0.001)^{*}$ & $0.463(<0.001)^{*}$ & $0.461(<0.001)^{*}$ \\
\hline DBP & $0.283(<0.001)^{*}$ & $0.372(<0.001)^{*}$ & $0.350(<0.001)^{*}$ \\
\hline
\end{tabular}

*= Statistically significant 